\title{
An Algorithm of Missile Incoming Area in Surface Warship Air Defense Operations
}

\author{
LONG Fei ${ }^{a}$, KAN Yabin and DENG Ke \\ Institute of Software and Simulation, Dalian Academy, Dalian, Liaoning, China
}

\begin{abstract}
Aiming at the algorithm of the enemy missile incoming area in surface warship air defense operations, this paper sets up the algorithm coordinate system, and distinguishes the position situation of both parties. Based on these, the paper defines the regional shape of the missile attack area, and establishes the calculation method. At last, it develops a demonstration verification program and supplies the application examples in various cases. The missile attack area can be calculated accurately with this algorithm, so it is able to effectively assist the commander to form scientific air defense plans.
\end{abstract}

\section{Introduction}

In surface warship air defense operations, the main air threats for our surface warship are from the incoming ship-to-ship missiles launched by surface warships, submarine-to-ship missiles launched by submarines, and shore-to-ship missiles launched by coastal missile forces. In response to the threat, our party may adopt the following air defense operations mode: Resisting the attack ship-to-ship/ submarine-to-ship/ shore-to-ship missiles with regional or close range air defense ships. This paper focuses on determining the enemy missile attack area, which is a key issue for air defense modes. The enemy missile attack area refers to the possible flight area for the enemy missiles when using ship-toship/ submarine-to-ship/ shore-to-ship missiles to carry out attacks. Our party must accurately grasp the enemy missile attack area as preplanning the air defense and making emergency decisions, so as to carry out effective defense demand analysis and force assignment, and form scientific resistance plans.

\section{Algorithm coordinate system}

Mastering the projection of the missile incoming area on the sea surface is adequate to formulate air defense operations plan in practice, so the plane rectangular coordinate system $x O y$ is established. The initial condition is that the location of our surface warship is Point $W$, and the enemy surface warship/submarine/coastal missile force locates in Point $O$, with a distance of $d$ in between. The enemy missile's maximum firing range is $R$, and the minimum distance of the terminal route is $r$. As is shown in Figure 1, a plane rectangular coordinate system can be set up with $O$ as the original point and $O W$ as the Axis $x$ forward. Let's rotate $O W 90$ degrees counter clockwise and get Axis $y$ forward. The Circle $\Omega_{1}$ is the enemy missile attack circle, with the center $O$, and the radius $R$; the Circle $\Omega_{2}$ is the attack circle of the missile's terminal route, with the center $O$, and the radius $r$.

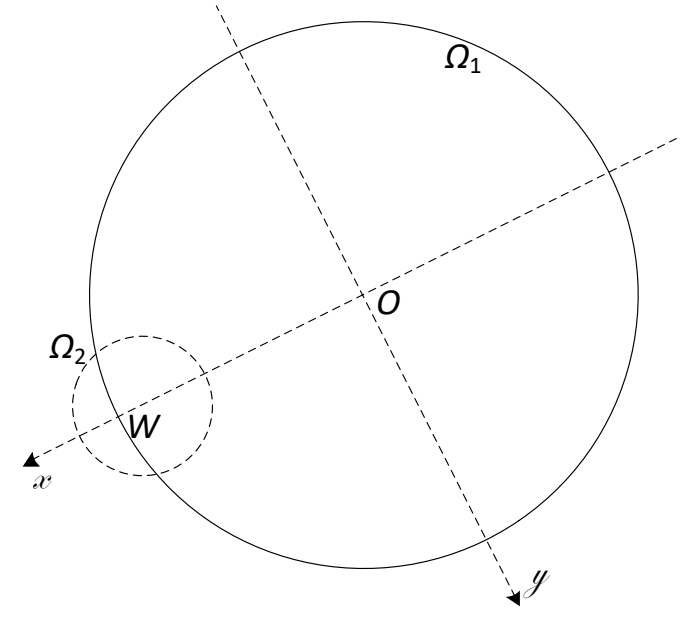

Figure 1. Algorithm coordinate system.

\section{Auxiliary ellipse and position situation}

When the enemy launches ship-to-ship/ submarine-toship/ shore-to-ship missiles to attack our surface warship, the missile incoming area gets to the maximum on condition that the enemy missile's route is programmed to have one turn. Therefore, the auxiliary Ellipse $P$ is established, the focus is $O, W$, and the length of the long axis is $R$. Make a vertical line of $O W$ passing Point $W$, and Point $A_{1}$ and Point $B_{1}$ are the intersections of the vertical and Ellipse $P$. Point $C$ and Point $D$ are the intersections of Ellipse $P$ and Axis $y$. Most part of the boundary of the enemy missile incoming area is on Ellipse $P$. With the changes of the distances of both parties, the shape and algorithm of enemy missile attack

*Corresponding author: doubao.0123@aliyun.com 
area are divided into two cases: Case 1, $\left|A_{1} B_{1}\right|<2 r$; Case2, $\left|A_{1} B_{1}\right| \geq 2 r$.

\section{Algorithm of missile incoming area}

\subsection{Case1 $\left(\left|A_{1} B_{1}\right|<2 r\right)$ algorithm}

\subsubsection{Description of the missile incoming area}

As in Figure 2, the auxiliary Ellipse $P$ in Case 1 is established. Point $A$ and Point $B$ are the intersections of Ellipse $P$ and Circle $\Omega_{2}$.

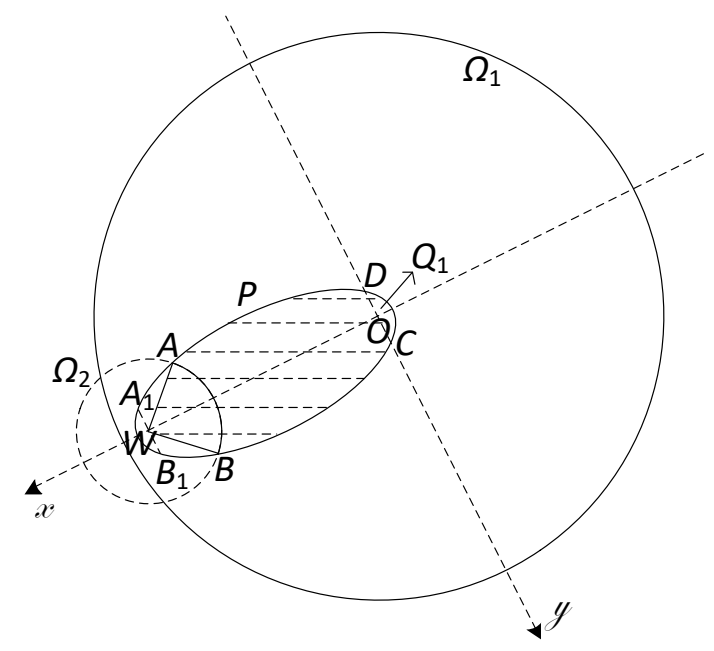

Figure 2. Missile incoming area in Case 1.

The shadow part in Figure 2 is the missile incoming area $L_{1}$, which should meet the following conditions at the same time. First, being inside or on the Ellipse $P$. Second, the enemy missiles do not reverse the flight during the attack, that is, Area $Q_{1}$ is excluded. Third, the distance of the enemy missile's terminal route is not smaller than $r$. Therefore, $L_{1}$ is:

$$
L_{1}=P-Q_{1}-\left(\Omega_{2} \cap P-S_{W A B}\right)
$$

Of which $Q_{1}$ is the area formed by the Segment $C D$ and the Arc $C D . S_{W A B}$ is a Sector, with Point $A$ and Point $B$ as the extreme points of the arc, and Point $W$ as the center.

Therefore, establish the equations of Circle $\Omega_{2}$ and Ellipse $P$, and calculate the coordinates of Point $A$, Point $B$, Point $C$ and Point $D$, so that the missile incoming area $L_{1}$ can be obtained.

\subsubsection{Calculation of the missile incoming area}

I. Coordinates of Point $A$ and Point $B$

Equation of Ellipse $P$ :

$$
\frac{(x-d / 2)^{2}}{a^{2}}+\frac{y^{2}}{b^{2}}=1
$$

Of which: semi-major axis $a=R / 2$; semi-minor axis $b=\sqrt{a^{2}-(d / 2)^{2}}$.

Equation of Circle $\Omega_{1}$ :

$$
(x-d)^{2}+y^{2}=r^{2}
$$

Point $A$ and Point $B$ are the intersection points of Circle $\Omega_{2}$ and Ellipse $P$. The coordinates of Point $A$ and Point $B$ can be obtained from simultaneous equations of Circle $\Omega_{2}$ and Ellipse $P$ :

Point $A$ coordinates,

$$
\left\{\begin{array}{c}
x_{A}=\frac{d^{2}+R^{2}-2 R r}{2 d} \\
y_{A}=-\sqrt{(R-r)^{2}-\left(\frac{d^{2}+R^{2}-2 R r}{2 d}\right)^{2}}
\end{array}\right.
$$

Point $B$ coordinates,

$$
\left\{\begin{array}{c}
x_{B}=\frac{d^{2}+R^{2}-2 R r}{2 d} \\
y_{B}=\sqrt{(R-r)^{2}-\left(\frac{d^{2}+R^{2}-2 R r}{2 d}\right)^{2}}
\end{array}\right.
$$

II. Coordinates of Point $C$ and Point $D$

Point $C$ and Point $D$ are on the Ellipse $P$. The abscissa of Point $C$ is $x_{C}=0$, and the abscissa of Point $D$ is $x_{D}=0$. Place $x_{C}=0, x_{D}=0$ into the equation of Ellipse $P$, and the coordinates of Point $C$ and Point $D$ can be obtained:

Point $C$ coordinates,

$$
\left\{\begin{array}{c}
x_{C}=0 \\
y_{C}=b \sqrt{1-\left(\frac{d}{2 a}\right)^{2}}
\end{array}\right.
$$

Point $D$ coordinates,

$$
\left\{\begin{array}{c}
x_{D}=0 \\
y_{D}=-b \sqrt{1-\left(\frac{d}{2 a}\right)^{2}}
\end{array}\right.
$$

\subsection{Case $2\left(\left|A_{1} B_{1}\right| \geq 2 r\right)$ algorithm}

\subsubsection{Description of the missile incoming area}

As in Figure 3, the auxiliary Ellipse $P$ in Case 2 is established. The text of your paper should be formatted as follows: 


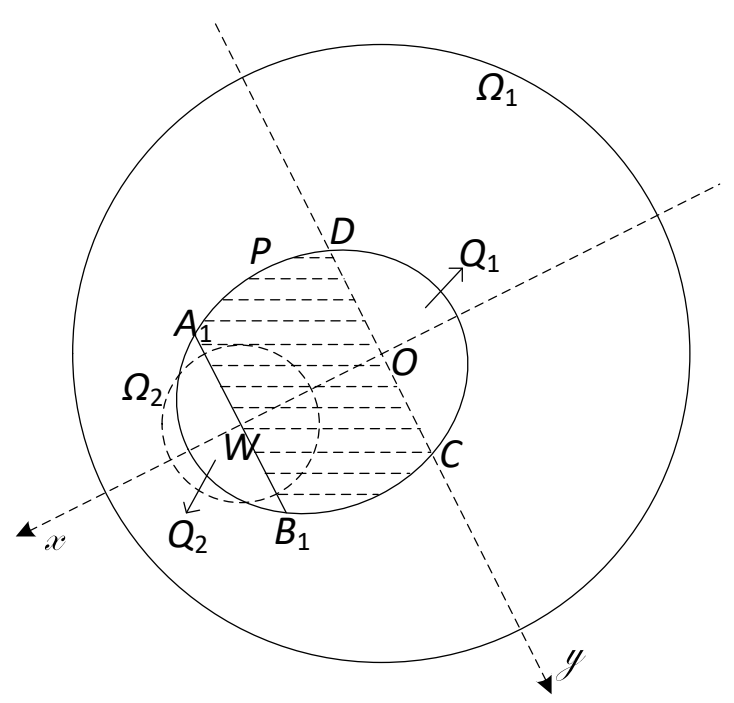

Figure 3. Missile incoming area in Case 2.

The shadow part in Figure 3 is the enemy missile incoming area $L_{2}$, which should meet the following conditions simultaneously. First, being inside or on the Ellipse $P$. Second, the enemy missiles do not reverse the flight during the attack, that is, Area $Q_{1}$ is excluded. Third, the area $Q_{2}$ is excluded. Therefore, $L_{3}$ is

$$
L_{2}=P-Q_{1}-Q_{2}
$$

Of which $Q_{1}$ is the area formed by the Segment $C D$ and the Arc $C D . Q_{2}$ is the area formed by the Segment $A_{1} B_{1}$ and the Arc $A_{1} B_{1}$.

The enemy missile incoming area $L_{2}$ can be obtained by calculating the coordinates of Point $A_{1}$, Point $B_{1}$, Point $C$ and Point $D$.

\subsubsection{Calculation of the missile incoming area}

I. Coordinates of Point $A_{1}$ and Point $B_{1}$

Point $A_{1}$ and Point $B_{1}$ are on the Ellipse $P$. The abscissa of Point $A_{1}$ is $x_{A_{1}}=d$, and the abscissa of Point $B_{1}$ is $x_{B_{1}}=d$. Place $x_{A_{1}}=d, x_{B_{1}}=d$ into the equation of Ellipse $P$, and the coordinates of Point $A_{1}$ and Point $B_{1}$ can be obtained:

Point $A_{1}$ coordinates,

$$
\left\{\begin{array}{c}
x_{A_{1}}=d \\
y_{A_{1}}=-b \sqrt{1-\left(\frac{d}{2 a}\right)^{2}}
\end{array}\right.
$$

Point $B_{1}$ coordinates,

$$
\left\{\begin{array}{c}
x_{B_{1}}=d \\
y_{B_{1}}=b \sqrt{1-\left(\frac{d}{2 a}\right)^{2}}
\end{array}\right.
$$

II. Coordinates of Point $C$ and Point $D$

Refer to the calculation formula of Case 1.

\section{Application example of the enemy missile incoming area}

In order to test the algorithm of enemy missile incoming area, a demonstration verification program is developed using Qt. It can be used to calculate the missile incoming area under various combinations of maximum range of the enemy ship-to-ship/ submarine-to-ship/ shore-to-ship missiles, the minimum distance of the terminal route, and distance between both parties.

Here is a set of assumed numeric values for calculating the missile incoming area in various cases. It is known that the position of our surface warship is Point $W$; the position of the enemy surface warship/ submarine/ coastal missile force is Point $O$; the maximum range of the enemy ship-to-ship/ submarineto-ship/ shore-to-ship missile $R$ is $520 \mathrm{~km}$. The minimum distance of the terminal route $r$ is $90 \mathrm{~km}$, corresponding to case 1 and case 2 . Assume that the distances between the enemy and our party $d$ are $480 \mathrm{~km}, 380 \mathrm{~km}$ and $300 \mathrm{~km}$, then the missile incoming area $L_{1}, L_{2.1}, L_{2.2}$, are calculated. The gray shadow areas in Figure 4-6 are the missile incoming area.

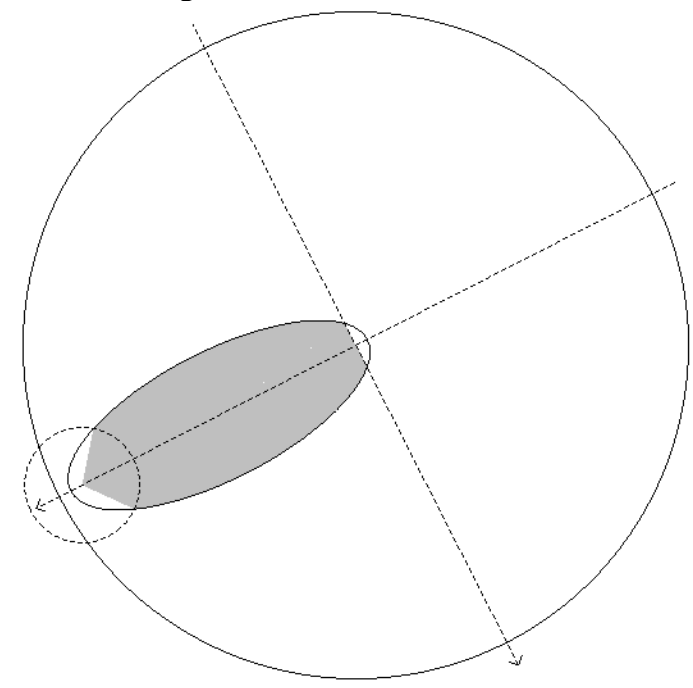

Figure 4. Calculation example of the enemy missile incoming area in Case $1(d=480 \mathrm{~km})$

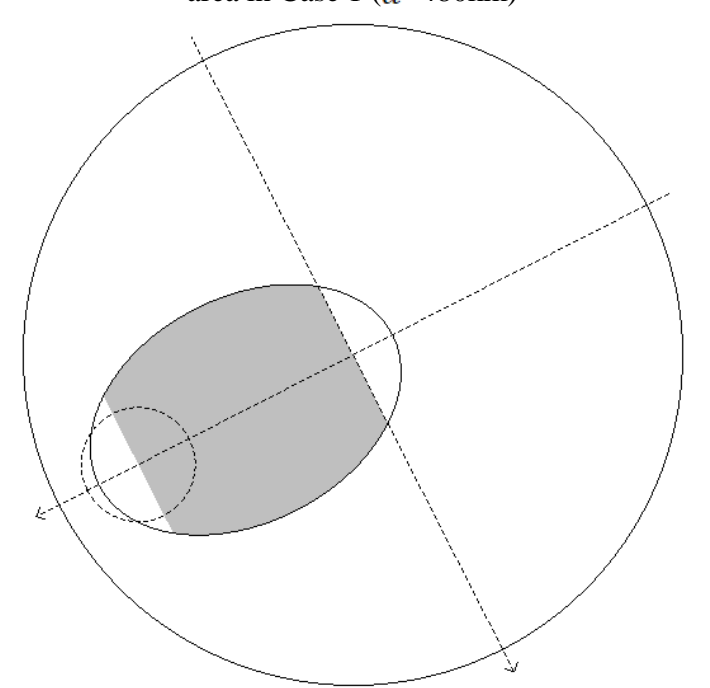

Figure 5. Calculation example of the enemy missile incoming area in Case $2(d=380 \mathrm{~km})$ 


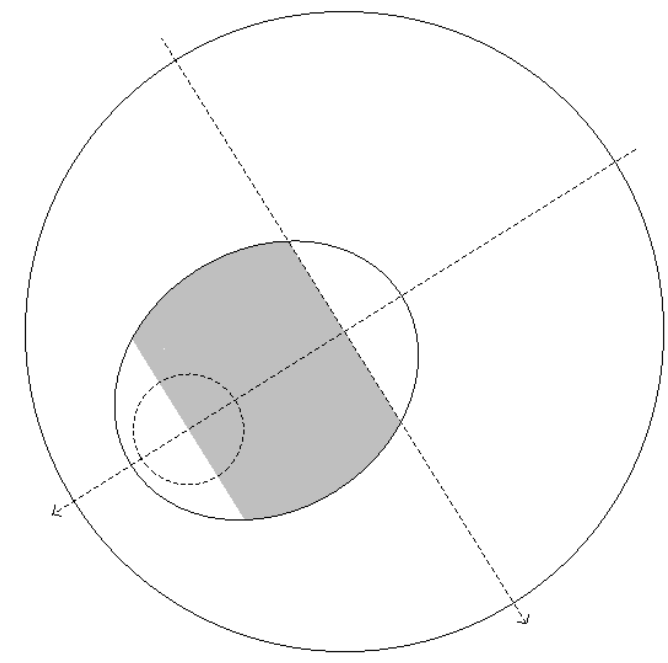

Figure 6. Calculation example of the enemy missile incoming area in Case $2(d=300 \mathrm{~km})$

\section{Conclusion}

Aiming at the algorithm of the enemy missile incoming area in surface warship air defense operations, this paper carries out military requirement analysis, algorithm coordinate system establishment and classification of position situation of both parties. On this basis, it describes the shapes of the enemy missile incoming area in various cases, and sets up the corresponding calculation model. Finally, a demonstration verification program is developed and the calculation examples in various cases are given. The algorithm put forward in this paper, which is able to accurately calculate the shape of the enemy missile incoming area, can be directly used as the basis for the analysis of air defense operations requirement and the assignment of forces. Besides, this algorithm will become an important function point of the operational planning software to effectively assist the commander to form scientific air defense plans.

\section{References}

1. XIE Xiaofang, SUN Tao, OUYANG Zhonghui, Anti-Ship Missile Route Planning Technology(National Defense Industry Press, Beijing, 2010)

2. MIAO Xudong, Naval Fleet Cooperative Air Defense Mission Planning Theory and Application(National Defense Industry Press, Beijing, 2013)

3. ZHANG Zhongyang, Air Defense Antimissile Missile (National Defense Industry Press, Beijing, 2012)

4. YANG Lujing, CHEN Zhigang, LI Yu, The Theory and Application of Combat Decision-aid(National Defense Industry Press, Beijing, 2016)

5. YAN Fengxin, ZENG Quanren, ZHANG Zhiqiang, C++ GUI Qt4 Programming(National Defense Industry Press, Beijing, 2013) 